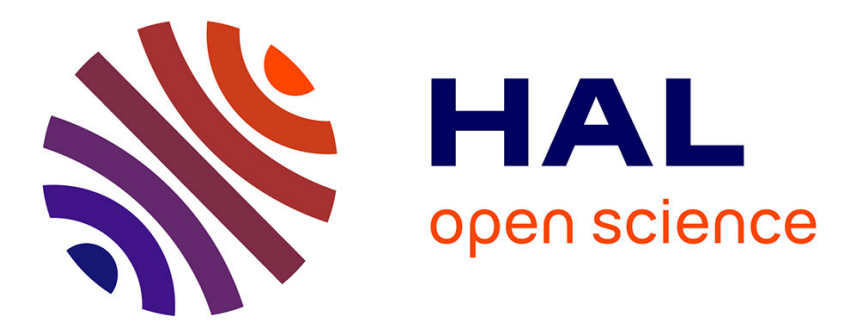

\title{
The Challenge of Designing Interactive Scenarios to Train Nurses on Rostering Problems in a Virtual Clinical Unit
}

Catherine Pons Lelardeux, Hervé Pingaud, Michel Galaup, Arthur Ramolet, Pierre Lagarrigue

\section{To cite this version:}

Catherine Pons Lelardeux, Hervé Pingaud, Michel Galaup, Arthur Ramolet, Pierre Lagarrigue. The Challenge of Designing Interactive Scenarios to Train Nurses on Rostering Problems in a Virtual Clinical Unit. Interactive Collaboratibve Learning, Sep 2018, KOS Island, Greece. hal-02127200

\author{
HAL Id: hal-02127200 \\ https://hal.science/hal-02127200
}

Submitted on 13 May 2019

HAL is a multi-disciplinary open access archive for the deposit and dissemination of scientific research documents, whether they are published or not. The documents may come from teaching and research institutions in France or abroad, or from public or private research centers.
L'archive ouverte pluridisciplinaire HAL, est destinée au dépôt et à la diffusion de documents scientifiques de niveau recherche, publiés ou non, émanant des établissements d'enseignement et de recherche français ou étrangers, des laboratoires publics ou privés. 


\title{
The Challenge of Designing Interactive Scenarios to Train Nurses on Rostering Problems in a Virtual Clinical Unit
}

\author{
Catherine Pons Lelardeux ${ }^{1}$, Herve Pingaud ${ }^{2}$, Michel Galaup ${ }^{3}$, Arthur \\ Ramolet $^{4}$, and Pierre Lagarrigue ${ }^{5}$ \\ 1 IRIT, University of Toulouse (France), INU Champollion, Serious Game Research \\ Network \\ catherine.lelardeux@univ-jfc.fr \\ 2 LGC, University of Toulouse (France), INU Champollion, Serious Game Research \\ Network herve.pingaud@univ-jfc.fr \\ 3 EFTS, University of Toulouse (France), INU Champollion, Serious Game Research \\ Network michel.galaup@univ-jfc.fr \\ ${ }^{4}$ SGRL, University of Toulouse (France), INU Champollion, Serious Game Research \\ Network arthur.ramolet@univ-jfc.fr \\ ${ }^{5}$ ICA, University of Toulouse (France), INU Champollion, Serious Game Research \\ Network pierre.lagarrigue@univ-jfc.fr
}

\begin{abstract}
The healthcare institutions in leading countries have undergone numerous changes to control their charges. This transformation generates an incredible impact on the organization of work specially for caregiving staff. At the same time, there has been an increasing interest for healthcare training in immersive training environments which could represent with great fidelity a professional context. Designing such a virtual environment allows to experiment real-life like complex situations without any risk for the inpatients. This research aims to create a digital and entirely controlled training clinical unit where future nurses could improve their task organization skills, situation awareness, decision-making and leadership. In this article, we present a method to design educational interactive scenarios to train them to plan their activity, deliver and organize the care for some fifteen inpatients. Then we build a dozen of scenario using this method. The trainee can freely act and make decision which could cause delay or equally bad and even far worse inadequate caregiving. Well-known in operation research as the nurse rostering problem, the trainee can experiments such realistic regular situations as well as non regular unexpected ones where irregularities or hazards occur and force them to revise their revise their projected schedule to the moving context.
\end{abstract}

Keywords: Virtual Environment, Serious Game, Nurse Rostering, Nurse training 


\section{Context}

All over the world and especially in healthcare institutions located in leading countries, the past decade has seen the rapid development of outpatient treatment in a day-care center. Nowadays, most of hospitals offer ambulatory services that would enable them to pool resources. As a consequence, professionals have to face to an increasing number of patients in shorter caregiving period. This situation mainly impacts the activity of healthcare teams who need to adapt and use a new workflow. Unlike other organizations, hospital staffs work $24 / 24 \mathrm{~h}$ and seven days a week with an increase of time pressure. Getting such systems under control requires management of delivered services. In spite of the quality of the management, the caregiving teams have to face to situations where unpredictable events can occur at any time. They must maintain the quality of service considering the level of uncertainty to which the healthcare teams are naturally exposed. As a consequence, irregular shift work have a real impact on the level of nurses well-being, contentment and therefore on the quality of care for the patients.

For many years, the research has tended to focus on scheduling and rostering problem by proposing algorithms to help organization to solve their individual short-term scheduling problems. The progress in planning and scheduling has not delivered widely adopted methods, algorithms and software tools as the underlying problems are really complex ones. Here, we plan to develop a virtual training environment to support nurse-students' teachers, as well as experimented nurses in a continuous education perspective, to develop non-technical skills as work organization, decision making, situation awareness.

\section{State of the art}

Since few years, healthcare professionals and trainers became interested in both inter-professional skills [3] and non-technical skills [11][10][1]. Globally, on one hand, there is a growing agreement regarding what should be assessed and on the other hand, many reports and experts point the importance to design educational environment and educational programs to reproduce with high fidelity the professional environment. It mainly relates to create experiential learning courses to foster students to develop inter-professional skills (both technical or non-technical skills). Specht and Sandlin[6] believe that "experiential learning focuses on 'doing' in addition to the 'hearing' and 'seeing' that occur in traditional lecture class". They also argue that experiential learning is a structured activity in which material and principles encountered are integrated and applied to new situations.

Most of training in healthcare which takes place in a digital world are designed for medical professionals and logically focused on technical skills and surgery [4]. A few ones are designed to train caregiving teams for better coordination and efficiency inside their operative unit[2][6]. Kilmon et al. target speed and accuracy of nurse response in emergency situations requiring cardiopulmonary resuscitation. Some of them intends to represent the complexity 
of the hospital supply chain by modeling the healthcare logistics using virtual RFID configuration. This is the case of Thomson et Hagstrom [12] who use Second Life as a support. Others have represented in a virtual operating room the inter-professional team activity. This team is composed by an anesthetist, a surgeon, an operating nurse and an anesthetist nurse [8][5].

\section{Purpose}

Now, to the best of our knowledge, no digital educational environment proposes to train nurse to organize their daily tasks. Traditionally in Nurse Schools, teachers used to provide paper exercises working with small size case of studies (three or four patients) to challenge their students about the organization of a typical day. We can list some reasons why there are very few educational environments for care-giving teams: many constraints and difficulties restrict the development of this kind of training. The main constraints refer to the socio-technical environment itself, which is a complex and moving context. Consequently, recreating artificially the conditions of work in a clinical department where several dozens of patients are hospitalized is hardly impossible in a real educational place. On the other hand, operational research has shown that the nurse rostering problem is an NP-hard combinatorial problem. It is extremely difficult to efficiently solve real life problems ex-ante, because of their size and complexity. Therefore, it should be easier to teach best practices in a controlled and well-defined system and to address the problem online in a secure and high fidelity context.

This research takes part of a global research that aims to provide a Real-time Digital Virtual Environments to train future nurses on scheduling their activity, providing and adapting their tasks to a moving context. This interactive environment is exclusively designed for educational purpose and provides experiential training situations where the student plays the role of a regular nurse in a clinical service.

This article presents a method to design educational scenarios which aims to support trainers to teach nurses the best practices to organize their daily tasks facing to an increasing number of patients, to evaluate the importance of unpredictable events and all types of disturbances, to adjust their projected schedule, to face to a set of uncertainties relative to the tasks that have to be performed in an inter-professional and moving context. All the scenarios designed with this method will be available in this innovative digital and immersive environment.

\section{Approach}

\subsection{Educational interactive scenario}

In the field of serious games, a scenario can be considered as a set of elements:(1) a briefing (mission): presentation of the current situation and expected objectives to reach, (2) a virtual universe: objects, furniture, documents, characters...(3) a set of actions, pieces of information, documents, furniture and 
objects which can be manipulated through the universe to achieve the mission, (4) playful and educational locks such as educational prerequisites, educational failures to avoid...(5) educational skills to develop or acquire, (6) abstract or concrete concepts which can be manipulated with interactivity through the environment: game play elements as inventory of assets, monetary system, virtual store... and educational concepts as programming, making decision...(7) steps or levels which compose the mission, (8) educational objectives to reach (visible or not in a briefing stage) (9) a debriefing: summary of outcomes with feedback that should help the player to succeed in the future.

Firstly, a scenario proposes to the players a short storytelling of what is the actual situation and what is the expected situation at the end. Secondly, a scenario provides interactions that allow the players to achieve the mission and locks (educational locks or playful locks) to prevent the player to succeed. Finally, outcomes are compared to expected objectives and results are immediately displayed at the end of a game session.

This definition particularly suggests that interactive storytelling triggers challenging opportunities in providing effective models for enforcing autonomous behaviors for characters. In other words, players should be able to be wrong, fix their errors, succeed or fail.

In the case of training environment for high graduated students, the classical challenge to design an interactive scenario consists in either (1) representing with creativity but also with high fidelity the professional environment through the virtual universe or (2) providing opportunities to characters to choose their own strategy, or (3) providing interactions as part of the professional activity using objects/equipment/furniture/abstract elements arranged in the virtual universe, or (4) giving relative but controlled freedom to act in the universe in order to compare with the expected behaviors.

Three kinds of scenario can be listed: - entirely controlled scenario: a script defines every possible path to succeed or fail the mission. - controlled scenario with a limited but real freedom: a large combination of paths are possible to succeed or fail. As a consequence, none script defines all the possible combinations but algorithms can calculate them if necessary. - entirely free scenario : machine learns from user's interactions and builds a statistically-realistic behavior. At the beginning, paths are unknown but machine learns from the user's experience over the time.

Designing an entirely controlled scenario consists in determining in details every available alternative and their consequences in the virtual world. Such a scenario is called scripted or branching scenario. It can be graphically represented by a tree-like structure. Our research intends to design a set of controlled and non-linear educational interactive scenarios. All possible combinations are definitely not predetermined even if a list of constraints must be set reduces the solutions space considering the domain rules. The most important for us is to compare the behavior of the nurse with what is expected. Models based on constraints [9] do not focus on the sequence of actions realized by the learner. It focuses only on results comparing the expected results to the learner's results. 
The system checks if all the constraints have been satisfied. To that end, a large freedom of action is given to the trainee respecting the rules of their professional activities.

\subsection{An educational virtual clinical service}

Our research intends to design a real-time digital environment, which represents a clinical department in a virtual hospital, individual organization as well as interaction with their colleagues and includes embedded monitoring tools to control nurses activity. It aims to transfer know-how from experienced nurses to junior by experiencing real-life situations in a virtual controlled and safe educational environment. Designing a virtual environment with a large library of well-known professional situations should support trainers to educate future nurses on task scheduling, situation awareness, leadership and decision-making.

The virtual environment represents all the actors with whom the regular nurse is used to cooperate such as the care giving staff and their patients. The staff can be composed of a head nurse, a caretaker, a doctor, a kinesiologist, courier from blood analysis laboratory, hospital porter... The trainee plays the role of a regular nurse who has to organize their working day and take care about their patients. This digital environment is designed with game mechanisms and interactive features such as a scheduling system, a task shifting and a decisionmaking system.

The trainer can choose an educational scenario from a library composed of various real-life ones. The nurse-student plays the role of a regular nurse and must manage the situation. The library of scenarios must be composed of regular situations as well as complex situations in which deficiencies or unpredictable events can occur or mistakes can be made and fix. Designing educational scenarios for scheduling training is particularly complex because most of the time, the causal chain of events that leads to change the initial formulation of a rostering problem is unpredictable. It implies a large variety of contributing factors, such as human factors, technical failures, patients pathologies... which are difficult to combine artificially.

\section{Method}

The method aims to structure the design process to potentially build a large number of educational scenarios with a large variety of professional situations. The main goal is to build a library of educational situations from real professional situations that occurred in French hospitals.

The method is composed of three stage: the domain analysis, the human activity modeling and the scenario (see fig. 1). The domain analysis stage allows to obtain information about the professional domain such as a typical clinical service organization, the average number of patients in a real one, inpatients, patient records, the composition of a medical staff, the care giving staff typical 
activities... It aims to highlight the concepts, the terminology, papers and documentation, the software and tools used... This step allows to collect elements to describe the virtual clinical service and a set of global professional activities which the care giving staff deals with in a real hospital.

The human activity modeling stage consists in structuring data to describe the universe and all elements that compose an interactive scenario.

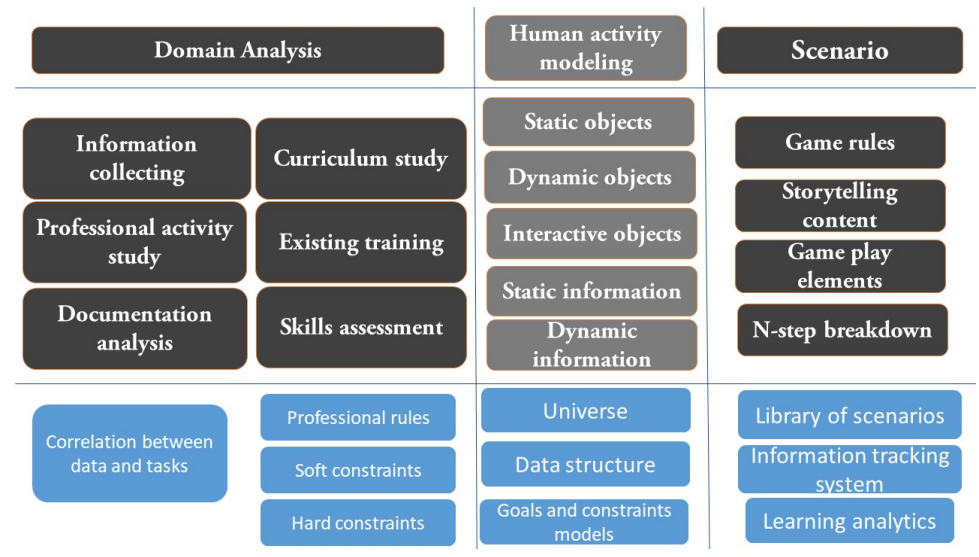

Fig. 1. The method is composed of three global steps: the domain analysis, the human activity modeling and the scenario scripting.

\subsection{Domain Analysis}

This step consists in interviewing experts, trainers and professionals to gather substantive information about the domain that will have implications for the assessment. Approximately some twenty semi-structured interviews have been conducted with a dozen of care experts and trainers who work either in Nursing Schools either in the Regional Healthcare Agency. Two dozen of experienced nurses have been interviewed and involved in the game design process. They were chosen because of their affiliation in eighteen different French hospitals. These criteria ensure to obtain a global consensus to represent a typical clinical service.

Real-life nurse's activity The analysis of real situations related to the targeted skills enables us to identify tasks and patient records with a gradual complexity. For example, an educational scenario must deal with a shift type which is defined by a start and end time. It should begin either early: 6:30-14:30, late: 14h30-21:30, or night 21:30-6:30. The scenario must comply with a typical daily scheduler. The typical day is split into main stages into which similar tasks are performed for all patients. Some are not hardly subject to constraints from the environment, some are not. For example, the delivery of breakfast is planned in 
the early morning just after team transmission from night to morning staff. The constraint is the availability of food trays that is generally satisfied in due time. However, the doctor in charge of the unit is making the visit of patient in the middle of the morning. This is considered as a hard constraint for two reasons. First, the doctor is often having a heavy workload including solicitations from emergencies or delay during surgical operations. It is likelihood that the visit will not be done at the expected time. Moreover, during the medical visit, doctors change the medical content of the Electronic Health Record (EHR) to adapt a therapeutic treatment or ask for new examinations (biological analysis, medical imaging). For the nurse, it means that the set of task has to be revised and the projected schedule will be impacted for the remaining part of their service. This typical day description revealed both the existence of generic activities and a predefined time slicing that punctuates the work flow. As an example, when the nurses begin and end their shift, they must listen and communicate with the previous/following giving care team to obtain/broadcast the most important information. The medical visit occurs at 10:00 am and the blood analysis must be done before 10:00 am if the results must be broadcast to the doctor before 14:00 am. All these substantive information allows to model a generic frame for a scenario and represent a typical clinical service.

Health records of real patients The second step relates a detailed analysis of a set of real patient records and associated activities (nurse's activity, nurse assistant's activity, doctor's medical visit...). Fifteen health patient records of real patients from a dozen of different hospitals has been analyzed and compiled. This analyzes has led to a standardized model of health patient record. A EHR is composed of three files: (1) an administrative file which contains administrative information about the patient, (2) a medical file which contains medical information about the health state and the health history, (3) a nurse file which contains care giving recommendations written by the previous nurses who gave health care to the patient.

Preliminary communications between experts lead rapidly to the conclusion of the diversity of representation of what health patient records are. It is a critical point as the nurse's tasks are daily reported and stored in files on the basis of the information delivered by the institution and by the doctors. The group of experts spent a long time to converge towards a unified and standardized model of a patient record. Differences concerned substantive issues on the semantics to use and the pieces of information to dispatch between the three files. If a rationalization of the EHR content has been reached, what we could call a convergence process of the expert views has really contributed to share a conceptual representation of the game design requirements and to strengthen the relationships between team members. As experts reached an agreement on a standardized patient record, they extracted valuable pieces of information from those 15records and lighted content about effective patients care needs as a key of educational purposes. At the end of the step, 15 virtual patient records have been designed (see fig.2).

Nurse's activity and constraints This step consists in understanding the rules and the cognitive scheme the experienced nurses use to organize their job. 


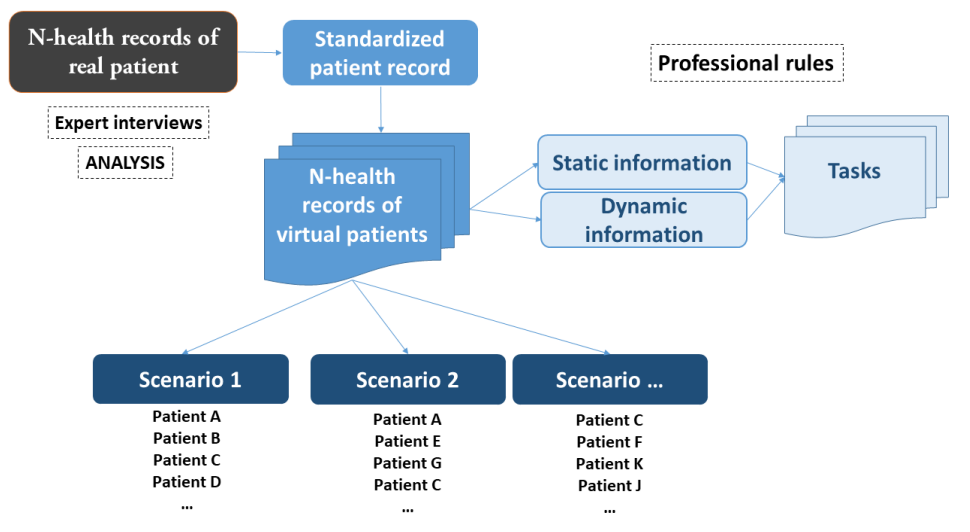

Fig. 2. A standardized health record results from the analysis of health records of real patients.

Nurses globally decide of their activity from last pieces of information orally delivered by the night shift and the EHR. The starting point to understand how they proceed to organize their activity was to analyze how they schedule their activity with the 15 virtual patients studied in the last stage. Manual scheduling of 15 patients care plans is an expensive time consuming task, even for experienced nurses. However, it really allowed us to understand their way of thinking and the rules they used to comply with professional excellence. Analyzing their approach on self-scheduling facing this virtual workload of 15 patients, we noticed that they proceed on a step-by-step trial and error process. First, they mentally compare the specific cares a patient needs with a standard well-known scheduling driving what could be named a generic framework of daily nurses activity. Secondly, they refresh this generic framework by task addition or/and task removal onto the timetable until they obtain a projected scheduling. Moreover, a feedback of our experience states that most of the time, nurses plan their activities using adjustments to a standard predefined planning after having performed a detailed analysis about the inpatient records.

An example of a task order practice is a breakfast postponing because the nurse must take a blood sample in fasting condition. Decision making is based on a kind of mimicry that is usually practiced in critical environments where service are delivered to people and affect their life.

The last step consists in balancing the workloads for the new set of tasks in a global perimeter with respect to all the rostering constraints: coverage constraints, time related constraints, hard constraints, soft constraints... Coverage constraints are constraints that refers to the number of professional roles needed in a working period. Time related constraints are constraints that refer to the scheduling, the right order of care delivered as well as other more personal activities. For example, personal lunch time, medication distribution time, patient lunch time, patient therapeutic care...Hard constraints are constraints that 
must be satisfied whatever event occurs. For example, give patient their medications. Soft constraints are constraints that are considered as recommended but they may relaxed if necessary to find a feasible solution.

A cross analysis of real-based situations and curriculum At this step, we need to identify and classify the knowledge and skills which are concerned by the studied patient records. It implies a cross analysis on the curriculum of Nursing School and the EHR of the virtual patients. For Le Boterf[7] 'the skill is the mobilization or activation of several knowledge, in a given situation and context'. This cross analysis work was carried out in collaboration with trainers from Nursing Schools and didactic researcher. In the repository of activities and skills for a graduate nurse, we find that the university program involves ten skills. Among these ten skills, the skill number 9 entitled "Organize and coordinate care interventions" is the one we mainly target. We are going to propose an answer to the needs of trainers through the use of a serious game in initial training. But, how is it possible to ensure that the skill is mastered? In other words, we must provide to the designers the criteria to evaluate the students. The referenced french national book details the activities and skills which must be assessed to be a graduated nurse. It mainly points three criteria. The first one concerns the relevance of the intervention of the various participants. The second is about the Consistency in the continuity of care. And the third one relates to the reliability and relevance of the information provided. Each indicator is associated with another one which gives visible signs. Let's take for example the criterion named Consistency in the continuity of care. The indicators relating to this criterion are: (1) the links between the various professional interventions are identified and well defined, (2) the organization of the activities in order to optimize collaborative work is explained and argued, (3) the control of the assigned care is done, (4) continuity and traceability of care is ensured.

This collaborative work of skill identification make the designers know what interactions must be monitored to assess the student at the end of a training session.

\subsection{The human activity modeling stage}

Representing the nurse's activity in a clinical unit implies to characterize a set of virtual patients, a set of non-player characters like the nurse assistant, the doctor, the courier, the hospital porter... who daily interacts with the nurse, a set of clinical rooms where the patients live, a nurse's room where the nurses cooperate, phone to other professionals and a room where nurses have a rest. We choose to define a virtual patient by all the pieces of information dispatched in their health record and all the pieces of information that can be delivered both by the patient itself and the others team members. The virtual patient model is exclusively based on the static information model and dynamic information model. The step of domain analysis showed that in average 40 pieces of information have been dispatched between the three files of the EHR and 40 tasks are associated to one virtual patient. The pieces of information are classify into two categories : a static information category and a dynamic information category. 
This choice of model enables us to create as much as we need new pieces of information whose can concern both virtual patients or anything else like 'Dr Vincent postpones the medical visit at 11:00'.

A static information is an information that can be read in the EHR whereas a dynamic information is an information that can only be delivered by a virtual character during a particular time period or after a task achievement. As an illustration, a patient calls the nurse to ease him, the patient family arrives in the clinical service and asks the nurse for an interview, during the medical visit, a doctor prescribes a new medicinal treatment or a new examination (radio, scanner, blood test...), the patient refuses to go with the hospital porter... These are some examples of dynamic information. They can be notified to the trainee at any time. These dynamic information are used to represent the moving context and enrich the story. In previous part, we described events that are known as having an impact on the planning during a typical day. To inform the trainee that the current context is changing, the dynamic information system can be used to notify an unexpected event. As an illustration, if the patient's health status is evolving either in a good way or in a bad way, the nurse must be informed and should be fostered to react very often.

There are two categories of tasks, the generic tasks which can be achieved on any virtual patients such as 'distributing breakfast and meal', 'making the bed', 'measuring temperature', 'measuring pulse rate'... and the contextual tasks which are associated with pieces of information dispatched in the universe. Most of the tasks are freely available since the associated pieces of information have been read or previous tasks have been realized. These kind of constraints allow designers to make a task available at any time or only when the constraints have been fulfilled.

\subsection{Scenario stage}

In terms of storytelling, designing educational scenarios based on real-life situations for professional training consists in two points. The first one consists in representing a perfect professional situation with competitive colleagues who realized all the prescribed tasks and do not postpone any care before the nurse begins their shift. The second one consists in representing a moving situation where medical prescriptions and unexpected events will occur around the clock and should foster the nurse to adapt their planning. If the nurse does not consider these new events in time, whatever their importance, problems should arise later or errors should be revealed as being part of the causal chain of events that could lead to an adverse event or a near miss. It should complexify the job for the healthcare team in charge of the next period. The models presented in 5.2 enable us to trigger new event that can disturb the nurse such as 'The family of patient A want to talk to you'. Another example can illustrate how to use a dynamic information to enrich the storytelling of a scenario. Non quality of logistics services around the clinical service could be a root cause of unexpected event for example. If a prescribed drug is not available in the hospital, the unit 
is not supplied and it could be critical for a patient. The nurse need to be informed that the hospital suffers from a lack of drugs. A dynamic information can be used to deliver a new drug prescription at 10:00 and another one can be created to inform the nurse that this drug is no longer available.

Each scenario is relative to the same clinical department with the option to choose the level of difficulty. The number of patients who need to obtain care is a parameter used to complicate a scenario. For example, a scenario addresses a group of 5 patients (A,B,C,D,E) while another one deals with 8 patients $(\mathrm{A}, \mathrm{C}, \mathrm{F}, \mathrm{G}, \mathrm{H}, \mathrm{I}, \mathrm{J}, \mathrm{H})$ and so on to propose a scenario in a unit of 15 inpatients (see fig.2).

\section{$6 \quad$ Results}

This method has enabled us to identify the main features and interaction systems needed to represent the nurse professional activity in a virtual world designed to train future professional on organization skills.

Using this method, it has been possible to design some ten scenarios which are all structured by five steps: (1)the briefing: to inform the students on their mission, (2)the communication step: to receive pieces of information from the previous staff and become aware of the situation when they shift at 6:30, (3)the scheduling step: to consult the patient's record and organize the activity of the day, (4)the activity itself: to provide care, organize medical examination, professional phone calls, patient discharge or arrival (5) the communication step: to inform the next staff on the current situation when they shift at 1:30pm During the domain analysis, we identified 6 features which would be part of game universe: (2) a virtual memory is attached to the nurse avatar. It should allow the trainees to use the pieces of information they read from the EHR or broadcast from other characters. It also stores a set of activities which can only be available since they become aware of particular information that are dispatched in the universe. This is the case of medical prescription for example. The task named 'Distributing per os treatment' is only available if they read the prescriptions on the medical patient record (2) an information reading system to hide some pieces of information which could be relevant to use as argument during a decision making stage, (3) a scheduler feature which allows the player to place and organize their tasks on their own scheduler from a typical daily scheduler, (4) a managing and delegating feature to allow the nurse to assign some tasks to the nurse assistant, (5) an argumentation feature to enable the student to express and argue their decision when they need to adjust the scheduler, (6) a communication system to enable the student to obtain/broadcast information to the previous/next caregiving team.

\section{Conclusion}

This paper aims to introduce a real-time digital environment designed to train the nurses to schedule and manage their activity in a complex and dynamic 
inter-professional context. We have first presented a method to design interactive and non-linear educational scenarios for self-planning nursing tasks. Then we have shown how the use of this method enabled us to build different scenarios combining a sub-group of virtual patients, all relevant for the training of nurses. Future work aims to realize training using this digital environment that embedded these scenarios in a dozen of National French Nursing Schools.

\section{Acknowledgments.}

These works are part of a global innovative IT program whose partners are University Champollion and the French Regional Healthcare Agency (Occitanie). The steering committee is composed of Ph.D. C. Pons Lelardeux, Ph.D.M. Galaup, Pr. H. Pingaud, Pr. P. Lagarrigue, C. Mercadier, V. Teihol.

\section{References}

1. Cornes, D.M.: Review of Interprofessional Education in the United Kingdom (19972013). Journal of Interprofessional Care 29(1), 85-85 (Jan 2015)

2. Petit dit Dariel, O.J., Raby, T., Ravaut, F., Rothan-Tondeur, M.: Developing the Serious Games potential in nursing education. Nurse Education Today 33(12), 1569-1575 (Dec 2013)

3. Gough, S., Hellaby, M., Jones, N., MacKinnon, R.: A review of undergraduate interprofessional simulation-based education (IPSE). Collegian 19(3), 153-170 (2012)

4. Graafland, M., Schraagen, J.M., Schijven, M.P.: Systematic review of serious games for medical education and surgical skills training. British journal of surgery 99(10), 1322-1330 (2012)

5. Hu, J., Feijs, L.: A Distributed Multi-agent Architecture in Simulation Based Medical Training. In: Pan, Z., Cheok, A.D., Mller, W., Chang, M. (eds.) Transactions on Edutainment III, pp. 105-115. No. 5940 in Lecture Notes in Computer Science, Springer Berlin Heidelberg (2009)

6. Kilmon, C.A., Brown, L., Ghosh, S., Mikitiuk, A.: Immersive virtual reality simulations in nursing education. Nursing education perspectives 31(5), 314-317 (2010)

7. Le Boterf, G.: valuer les comptences. Quels jugements? Quels critres? Quelles instances. Education permanente 135(2), 143-151 (1998)

8. Lelardeux, C.P., Panzoli, D., Galaup, M., Minville, V., Lubrano, V., Lagarrigue, P., Jessel, J.P.: 3d Real-Time Collaborative Environment to Learn Teamwork and Non-technical Skills in the Operating Room. In: Interactive Collaborative Learning. pp. 143-157. Springer, Cham (Sep 2016)

9. Ohlsson, S.: Constraint-based student modeling. In: Student modelling: the key to individualized knowledge-based instruction, pp. 167-189. Springer (1994)

10. Reese, C.E., Jeffries, P.R., Engum, S.A.: Learning together: Using simulations to develop nursing and medical student collaboration. Nursing education perspectives 31(1), 33-37 (2010)

11. Riem, N., Boet, S., Bould, M., Tavares, W., Naik, V.: Do technical skills correlate with non-technical skills in crisis resource management: a simulation study. British journal of anaesthesia 109(5), 723-728 (2012)

12. Thompson, C.W., Hagstrom, F.: Modeling healthcare logistics in a virtual world. IEEE Internet Computing 12(5) (2008) 